PROCEEDINGS OF THE

AMERICAN MATHEMATICAL SOCIETY

Volume 126, Number 11, November 1998, Pages 3199-3200

S 0002-9939(98)04576-6

\title{
INTEGER SETS WITH DISTINCT SUBSET SUMS
}

\author{
P. E. FRENKEL
}

(Communicated by David E. Rohrlich)

\begin{abstract}
We give a simple, elementary new proof of a generalization of the following conjecture of Paul Erdős: the sum of the elements of a finite integer set with distinct subset sums is less than 2 .
\end{abstract}

Let $a_{0}<a_{1}<\cdots<a_{n}$ be positive integers with all the sums $\sum_{i=0}^{n} \varepsilon_{i} a_{i}\left(\varepsilon_{i}=0 ; 1\right)$ different. It was conjectured by P. Erdős and proved by C. Ryavec that then

$$
\sum_{i=0}^{n} \frac{1}{a_{i}}<2\left(=\sum_{i=0}^{\infty} \frac{1}{2^{i}}\right)
$$

(see [1]). F. Hanson, J. M. Steele and F. Stenger [2] proved the generalization

$$
\sum_{i=0}^{n} \frac{1}{a_{i}^{s}}<\frac{1}{1-2^{-s}}\left(=\sum_{i=0}^{\infty} \frac{1}{2^{i s}}\right)
$$

for all real $s>0$. These proofs are relatively simple but use generating functions and other methods in analysis. I have recently learned that a brilliant elementary solution to Erdős's original problem was found by A. Bruen and D. Borwein, more than 20 years ago. See [3] or [4].

We prove by elementary methods the more general statement that (continuing to assume that all sums $\sum_{i=0}^{n} \varepsilon_{i} a_{i}$ are different)

$$
\sum_{i=0}^{n} f\left(a_{i}\right) \leq \sum_{i=0}^{n} f\left(2^{i}\right)
$$

for any convex decreasing function $f$.

The hypothesis implies for $k=0 ; 1 ; \ldots ; n$ that

$$
\sum_{i=0}^{k} a_{i} \geq 2^{k+1}-1
$$

since there exist $2^{k+1}-1$ distinct positive integers (namely, $\sum_{i=0}^{k} \varepsilon_{i} a_{i}\left(\varepsilon_{i}=0 ; 1\right.$, $\left.\left.\left(\varepsilon_{i}\right)_{0}^{k} \neq(0)_{0}^{k}\right)\right)$ which are all less than or equal to $\sum_{i=0}^{k} a_{i}$.

Consider all $(n+1)$-tuples of positive integers $a_{0}<a_{1}<\cdots<a_{n}$ having property $(*)$ for $k=0 ; 1 ; \ldots ; n$. It suffices to prove that among all these, the $(n+1)$-tuple

Received by the editors April 7, 1997.

1991 Mathematics Subject Classification. Primary 11B13; Secondary $11 B 75$.

Key words and phrases. Sequences, subset sums.

The author thanks L. Laczkó for calling his attention to the problem, and M. Laczkovich for his attention and kind help.

(C) 1998 American Mathematical Society 
$a_{i}=2^{i}(i=0 ; 1 ; \ldots ; n)$ has maximal $\sum_{i=0}^{n} f\left(a_{i}\right)$. Consider any such $(n+1)$-tuple. We define an index $k$ to be good if equality holds in $(*)$ and bad otherwise. If all indices are good then clearly $a_{i}=2^{i}(i=0 ; 1 ; \ldots ; n)$. If not, then let $p$ be the smallest bad index. If there is any good index larger than $p$, then let $q$ be the smallest such index. Since $a_{i}=2^{i}$ for $i<p$ and $a_{p}>2^{p}$, it follows that the number $a_{p}-1$ is a positive integer and does not occur among the numbers $a_{i}$. If $q$ exists, then $q \neq 0$ and so

$$
a_{q}=\sum_{i=0}^{q} a_{i}-\sum_{i=0}^{q-1} a_{i}<2^{q+1}-1-\left(2^{q}-1\right)=2^{q},
$$

since $q-1$ is bad and $q$ is good. If $q \leq n-1$, then $a_{q+1}=\sum_{i=0}^{q+1} a_{i}-\sum_{i=0}^{q} a_{i} \geq 2^{q+1}$, hence $a_{q}+1<a_{q+1}$ and so the number $a_{q}+1$ does not occur among the numbers $a_{i}$.

Therefore, we may replace $a_{p}$ by $a_{p}-1$ and, if $q$ exists, $a_{q}$ by $a_{q}+1$. The property $1 \leq a_{0}<a_{1}<\cdots<a_{n}$ and the property $(*)$ will be preserved (this follows from the definition of $p$ and $q$ ). Since $f$ is decreasing and convex, the sum $\sum_{i=0}^{n} f\left(a_{i}\right)$ will not be decreased whether $q$ exists or not.

We may repeat this procedure until we reach the $(n+1)$-tuple $a_{i}=2^{i}$. This will happen after a finite number of steps since the sum $\sum_{i=0}^{n}(n+1-i) a_{i}$ takes only positive integer values and is decreased by at least 1 in every step. This completes the proof.

It is easily seen that if $f$ is strictly decreasing and strictly convex (as in the case $\left.f(x)=x^{-s}(s>0)\right)$, then equality in (1) holds only for $a_{i}=2^{i}(i=0 ; 1 ; \ldots, n)$.

\section{REFERENCES}

1. S. J. Benkoski and P. Erdős, On weird and pseudoperfect numbers, Math. Comp. 28 (1974), 617-623. MR 50:228; MR 50:12902

2. F. Hanson, J. M. Steele and F. Stenger, Distinct sums over subsets, Proc. Amer. Math. Soc. 66 (1977), 179-180. MR 56:5482

3. Canadian Mathematical Bulletin 17 (1975), 768, Problem P. 220.

4. R. Housberger, Mathematical Gems III, The Dolciani Mathematical Expositions, 1985, 215223.

KútVÖLgYi Út 40, BudAPEST 1125, Hungary

E-mail address: frenkelp@cs.elte.hu 